\title{
Effect of Educational Protocol about Resuscitation Process on Nurses' Performance
}

\author{
Randa Moustafa Ali(1), Jehan Sayyed Ali(2), Inshrah Roshdy Mohammed(3), Eman Fadel Abd Elkhalek(4).
}

1. Demonstrator of Medical Surgical Nursing (Critical Care Nursing), Faculty of Nursing - Minia University- Eygpt.

2. Professor of Medical Surgical Nursing Department, Faculty of Nursing - Minia University-Eygpt.

3. Assistant prof. of Medical Surgical Nursing Department, Faculty of Nursing - Minia University- Eygpt.

4. Lecturer of Medical Surgical Nursing, Faculty of Nursing - Minia University.

\begin{abstract}
Background: Cardiopulmonary resuscitation (CPR) is an emergency procedure for a person whose heart has stopped or no longer can breathing, CPR save live. Nurses' role is vital in resuscitation process. Aim of the study: to Evaluate effect of educational protocol about resuscitation process on nurses' performance. Research design: Quasi experimental research design utilized to fulfill the purpose of this study. Subjects: A Purposive sample included sixty nurses from critical care units. Setting: This study was carried out at five critical care units affiliated to Minia University Hospitals- Eygpt. Tools of data collection: Two tools designed and used for collecting data for this study, First: structured questionnaire sheet to assess nurses' knowledge, second: Observational checklist for nurses' practice sheet regard resuscitation process. Results: There was a highly statistically significant difference in nurses' knowledge and practice pre and post educational protocol regarding resuscitation process. Also, there was no statistically significance between personal data and their knowledge level or practice score. Conclusion: Based on the results of this study educational protocol can increase nurses' performance about resuscitation process. Recommendations: Greater attention and closely observation from hospital on nurse's performance during resuscitation process. Replication of the study should be done on a large sample.
\end{abstract}

Abstract

Keyword: cardiopulmonary resuscitation, nurses' performance, critical care nurses, educational protocol, resuscitation process.

\begin{abstract}
Introduction
Cardiopulmonary resuscitation (CPR) procedure as a basic skill is one of the most important inventions of the history in medicine with a rapid and urgent intervention to prevent or postpone death in a patient with cardiac arrest (Hasselqvist et al., 2015). CPR consists of an initial assessment of the patient and a subsequent number of steps that includes chest compressions, airway maintenance and rescue breathing (Yang, et al., (2012). CPR is one of the procedures that can be administered without an explicit order from a doctor which most incidences of cardiac arrest are both sudden and unexpected so, immediate actions are necessary (Waldron et al, 2016). CPR has been shown to reduce cardiac death in-hospital and related facilities when patients are managed by adequately trained health care professionals (Simmes et al., 2012). CPR is a medical practice for all cardiac arrests except where a "Do not resuscitate" (DNR) or "No CPR" order has been given for a particular patient ( Burkle et al., 2013). Nurse is a key role in respond to a cardiac arrest and initiate basic life support while waiting for the advanced cardiac life support team to arrive (Terzi, 2012). Throughout the years, as CPR guidelines are altered and the roles of the multidisciplinary team members are also subjective to change. So, Some of these new roles include: the rapid response nurse, the initiation of external defibrillation, the involvement in CPR decision making, chest compression, ventilation role, the understanding of the use of resuscitation drugs and the family support in the cases of witnessed resuscitation (Monsieurs et al., 2015). Nurses are the central part of the health care system and they are believed to be knowledgeable and competent in caring for patients.( Ray, 2016).
\end{abstract}

\section{Significance of the study}

Cardiac arrest is a major public health problem affecting thousands of individuals each year in both out-ofhospital and in-hospital settings (Hasegawa et al. (2013). In 2012 nearly 383,000 out-of-hospital sudden cardiac arrests

P a g e $\mid 2$ occurred and approximately 209,000 cardiac arrests occurred within a hospital. Survival rates from cardiac arrest are improving, however overall rates of survival are still low (American Heart Association, 2012).

Through my experience as a clinical instructor over a period of 2 years working in intensive care units I observed lack in nurses' knowledge and practice during resuscitation process that affect on patient's condition and delayed patient out. Therefore, this study designed in an attempt to improve nurses' performance during resuscitation process.

\section{Aim of the Study}

Evaluate effect of educational protocol about resuscitation process on nurses' performance.

\section{Research Hypothesis:}

The nurses' knowledge and practice about resuscitation process will be better than pre educational protocol.

\section{Study Design:}

Quasi - experimental research design was utilized to fulfill the purpose of this study

\section{Subjects:}

A purposive sample of about 60 critical care nurses who were working in a five Critical Care Units at Minia University Hospital at the time of conducting the study and willing to participate in the study was included.

\section{Inclusion Criteria:-}

- All nurses were in duties.

- Nurses who attended or not attended previous training courses about resuscitation process.

\section{Exclusion Criteria:}

- Nurses refuse participation in the study. 
Setting:

All Critical Care units affiliated to Minia University Hospital including (Intensive Care Unit, Emergency unit, Cardiothoracic Care Unit, Cardiac Care Unit, Stroke).

\section{Study Duration:}

The total data collection was collected for five months from February to July 2019.

\section{Tools for data collection}

Two tools were designed and used for collecting data for this study, these tools were formulated and tested by the researcher, and the content of the tools was established after extensive literature review.

\section{First Tool: "Structured Questionnaire Sheet" it consists of (two parts):}

1st Part: - Nurses' personal data to collect nurses' data related to the following (age, gender, years of experience, level of education ...etc.

2nd Part: - Knowledge assessment questionnaire sheet about resuscitation process and nurses' role in resuscitation team.it was multiple choice questions sheet translated into Arabic language.

\section{Scoring System:}

Each right answer takes one grade and the wrong answer take zero grade with a total scores 38 , so $(<60 \%)$ was considered unsatisfactory; $(\geq 60 \%)$ was considered satisfactory.

Second Tool:"Observational checklist for assessment nurses' practice in resuscitation Process"

This tool consists of (23) items for evaluating nurses' performance regarding their roles during resuscitation for critically ill patients in different critical care units.

\section{Scoring System:}

Each right answer (Done) take one grade and the wrong answer (Not done) take zero grade with a total scores 23 , so $(\leq 70 \%)$ was considered poor, $(>70-85 \%)$ was considered fair and more than $(85 \%)$ was considered good practice scores.

Educational Brochure about CPR Process. It was formulated by the researcher after extensive literature review and revised by experts in the field of critical care nursing. It was translated into the Arabic language with figures for more clarification.

\section{Tools validity:}

The study tools were developed after reviewing the related literature. To determine content and face validity, these tools were tested by a Jury committee that consists of five medical surgical-nursing experts. Recommendations were followed; the questions that were not appropriate were taken out and some questions needed clarification and modifications were done.

\section{Tools reliability:}

Tools were tested for content reliability using Alpha Cronbach's test. It was $(0.81)$ for the first tool, $(0.79)$ for the second tool.
Pilot study: A pilot study was carried out on 6 nurses working in the Critical Care units who fulfilled the inclusion criteria to test the feasibility, objectivity, applicability of the study tools, and to estimate the needed time to fill the data collection. Based on the results of the pilot study, no refinement/ modifications were done for data collection instruments; nurses who shared in the pilot study were included in the actual study sample.

Ethical consideration: Official permission to conduct the study was obtained from faculty ethical committee of research, dean of the faculty of nursing at Minia University, Research center afflicted to Egypt Ministry of Health, agreement from Egypt academic for the research center and technology and from directors of Critical Care Units at Minia 43 University Hospital. Oral consents were obtained from each nurse after explanation of the nature and purpose of the study. Each head nurses who were on duty during study implementation and nurses were free to either participate or not in this study and had the right to withdraw from the study at any time without any rational; also, nurses were informed that data will not be included in any further researches without another new consent. Confidentiality and anonymity of each subject were assured through coding of all data. (60\%) was considered poor, $(\geq 60-85 \%)$ was considered fair and more than $(85 \%)$ was considered good practice scores.

Study Procedure: Once official permissions were granted, the pre-educational data (pre-test) collected through (four weeks) to test studied sample for actual level of knowledge and practices regarding resuscitation process. The researcher distributed the Knowledge assessment questionnaire sheet to assess study sample' s actual level of knowledge after clearly explaining the way to fill it, then Observational checklist for assessment nurses' practice in resuscitation Process"

The total number (60) of the study participants was divided into twenty classes each class consists of three participants. The appointment for starting educational sessions was scheduled with the study sample according to their circumstances. The educational protocol was conducted as one session for knowledge teaching, two sessions for practice teaching for every class; each session took about 30- 45 minutes. At the end of teaching sessions (knowledge \& practice), the researcher gave the study participants brochure as an educational protocol about resuscitation process: It was formulated by the researcher after extensive literature review by experts in the field of critical care nursing , and translated into the Arabic language with figures for more clarification.

The researcher collects post educational evaluation (post-test) for knowledge through giving the sample the Knowledge assessment questionnaire sheet and Observational checklist.

as an immediate posttest assessment, then follow up was done by the researcher after three months by using $\mathbf{2}$ nd Part (Knowledge assessment questionnaire) \& Observational checklist .

To evaluate the effectiveness of the educational protocol regarding study sample' performance. Statistical analysis of data: Data obtained from the study tools were categorized, tabulated, analyzed and data entry was performed using the SPSS software (statistical package for social sciences version 22.0). Descriptive statistics were applied (e.g. 
Minia Scientific Nursing Journal (Print) (ISSN 2537-012X) Vol. (7) No. (1) June 2020

mean, standard 45 deviations, frequency, and percentage). Pearson's correlation coefficient was applied between quantitative variables. A significant level value was considered when $\mathrm{p}<0.05$. The smaller the P-value obtained, the more significant is the result $\left(^{*}\right)$, less than 0.001 was considered highly significant $(* *)$. The $\mathrm{P}-$ value is the probability of error of the conclusion.

\section{Results}

Table (1): Percentage distribution of the studied groups as regards personal data $(n=60)$.

\begin{tabular}{|c|c|c|}
\hline Personal data & No. (60 nurses) & $100 \%$ \\
\hline \multicolumn{3}{|l|}{ Age categories } \\
\hline - $20-24$ & 31 & 51.7 \\
\hline - $25-29$ & 7 & 11.6 \\
\hline - $\quad \geq 30$ & 22 & 36.7 \\
\hline Mean \pm SD & \multicolumn{2}{|c|}{$27.88 \pm 7.709$ years } \\
\hline \multicolumn{3}{|l|}{ Gender } \\
\hline - Male & 19 & 31.7 \\
\hline - Female & 41 & 68.3 \\
\hline \multicolumn{3}{|l|}{ Education Level } \\
\hline - Bachelor & 9 & 15 \\
\hline - Technical institute & 33 & 55 \\
\hline - Diploma \& secondary school & 18 & 30 \\
\hline \multicolumn{3}{|l|}{ Years of experience in ICU } \\
\hline - $\quad<5$ years & 36 & 60 \\
\hline - $\geq 5$ years & 24 & 40 \\
\hline Mean \pm SD & \multicolumn{2}{|c|}{$5.2667 \pm 2.655$ years } \\
\hline \multicolumn{3}{|l|}{ Previous workshop attendance } \\
\hline - Yes & 14 & 23.3 \\
\hline - $\quad$ No & 46 & 76.7 \\
\hline
\end{tabular}

Table (1) revealed that more than half of the study group's age was between 20-24 years and their Mean average age \pm SD was $(27.88 \pm 7.709)$ years and the highest percentage from them constituted $(68.3 \%)$ were females, also as regards their educational level results found that above the half among them had graduated from technical institute degree in nursing. On the other hand, the highest percentage of participants constituted $(60 \%)$ had less than five years of experience in working at critical units ,but lowest percentage of them were more than five years of experience and their Mean average \pm SD was (5.2667 \pm 2.655 years). Finally, as regards, previous workshop attendance regarding cardiopulmonary resuscitation process results revealed that the majority of the study group constituted (76.7\%) hadn't attended any previous workshop .

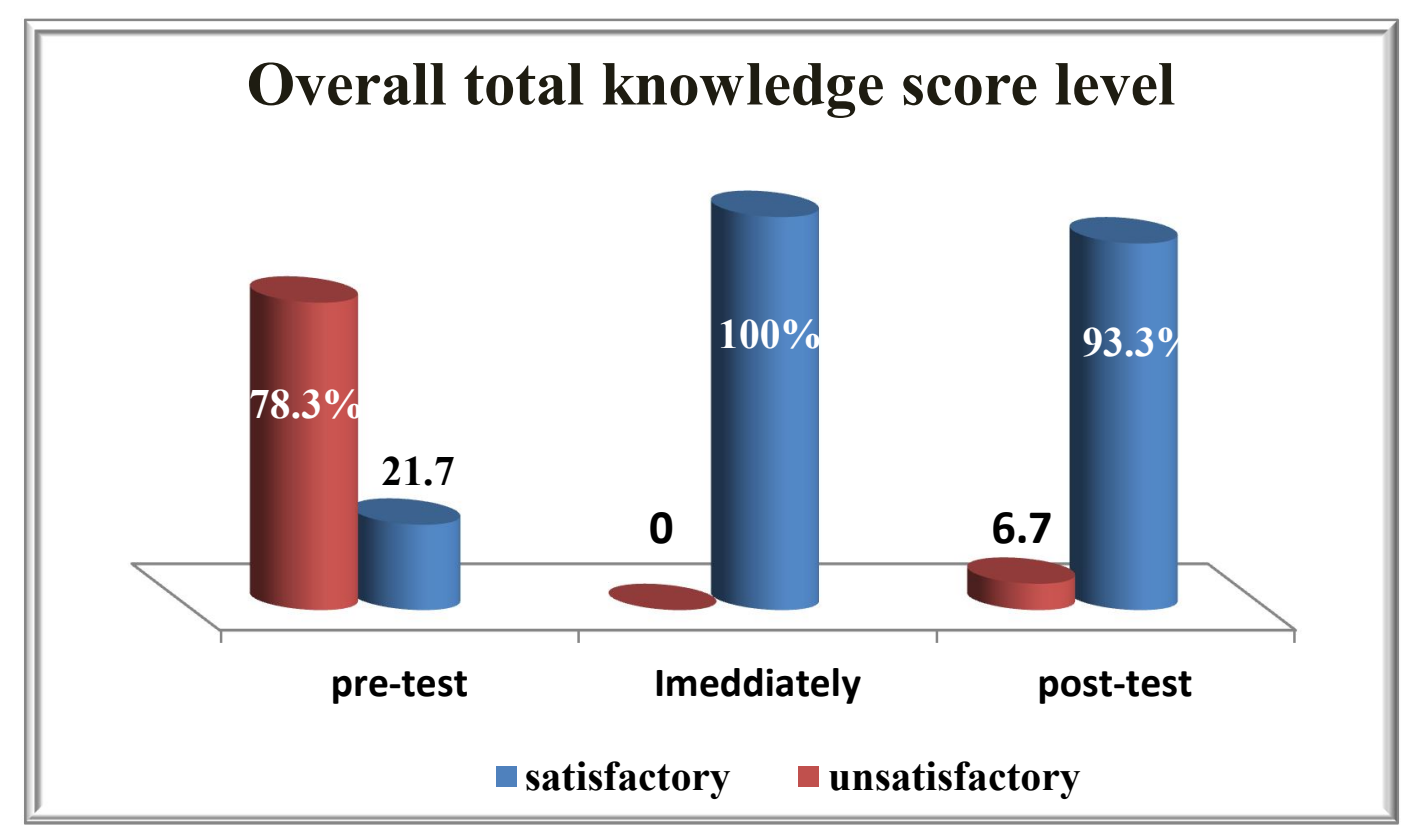

Figure (1): Overall total studied samples' knowledge levels about CPR process in the pre-post education

Figure (1) revealed that $78.3 \%$ of studied sample had unsatisfactory knowledge during pre education 5 while $100 \%$ of them had hundred percent knowledge levels immediately post education, while post education after three months $93.3 \%$ of them had satisfactory knowledge level. 


\section{Overall total practice levels}

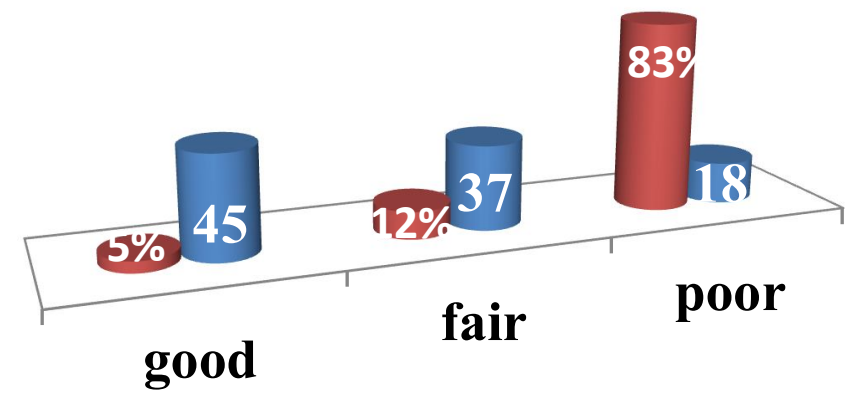

post-test pre-test

Figure (2): Overall total practice levels of the Studied groups in about CPR process through pre-post educational protocol l(three months)

Figure (2) displayed that $83 \%$ of study sample had poor practice level during pre test 'while $45 \%$ of them had good practice level post three months test.

Table (2) Correlations between total knowledge score and total practice score among studied sample pre\& post educational protocol

\begin{tabular}{|ccc|c|c|c|}
\hline \multirow{2}{*}{ Total Knowledge score } & \multirow{2}{*}{ Total practice score } & \multicolumn{2}{|c|}{ Pre - education } & \multicolumn{2}{|c|}{ Post - education } \\
\cline { 4 - 7 } & & $\mathbf{R}$ & $.016^{*}$ & -0.822 \\
\hline$\bullet$ & Pre- education & & 0.310 & $.000^{* *}$ \\
\hline$\bullet$ & Post education & & -0.136 & $.300(\mathrm{NS})$ & 0.618 \\
\hline
\end{tabular}

Table (2) showed that there was highly statistical significance between total knowledge and practice score at pre \& post educational protocol (three months).

$\underline{\text { Table (3) Correlations between personal data and total practice score at pre\& post educational protocol }}$

\begin{tabular}{|c|c|c|c|c|}
\hline \multirow{2}{*}{ personal data } & \multicolumn{2}{|c|}{ Pre- education } & \multicolumn{2}{|c|}{ Post-education } \\
\hline & $\mathbf{R}$ & p-value & $\mathbf{R}$ & p-value \\
\hline - Age categories & 0.063 & $.630(\mathrm{NS})$ & -.253 & $.052 *$ \\
\hline - Gender & -0.091 & $.487(\mathrm{NS})$ & 0.077 & $.560(\mathrm{NS})$ \\
\hline - Education level & 0.079 & $.550(\mathrm{NS})$ & -0.033 & $.800(\mathrm{NS})$ \\
\hline - Years of experience in working at critical units & -0.082 & $.534(\mathrm{NS})$ & -0.037 & $.776(\mathrm{NS})$ \\
\hline $\begin{array}{l}\text { Previous workshop attendance regarding } \\
\text { resuscitation process }\end{array}$ & -0.067 & $.611(\mathrm{NS})$ & -.047 & $.722(\mathrm{NS})$ \\
\hline
\end{tabular}

Table (3) reveled that there was no statistically significant correlation between all personal characteristics for studied groups and their total practice score pre and post-educational protocol except in a studied sample's age categories it had a significant correlation with mean practice score after three months post education with p-value $\left(052^{*}\right)$.

\section{Discussion}

Cardiopulmonary resuscitation (CPR) is an important medical procedure which is critically required for individuals who face sudden cardiac arrest or those who suddenly lose their consciousness) Ray, 2016). CPR knowledge and skill is extremely important to prevent and return the life of suddenly collapsed patient, so that nurse should become knowledgeable and skillful in delivering quality CPR (Wolfe et al., 2014).

During the current study the results of 60 nurses included in the study were have age from 20:24 years with the mean average age $\pm \mathrm{SD}$ was $(27.88 \pm 7.709)$ years; this may be due to shortage of the staff or the desire of university's hospitals to provide high-quality health care services, through employing as possible new graduates of the faculty of nursing or nursing technical institute in critical units.
Results of the current study were agree with (Elazazay et al., 2012) they conducted a study entitled effect of cardiopulmonary resuscitation training program on nurses knowledge and practice and they found that the highest percentage of the study group' age was between 20 to 25 years, Also, results supported by (Parajulee \& Selvaraj, 2011) who stated that the majority of the study sample age ranged from 20-25 years, In addition, a further validation by (Selman \& Ahmed, 2018) who stated that the half of the study group nurses who work in critical unites are with age range $18-24$ years old $(50 \%)$.

Findings of the present study showed that more than two third of the total study group were females. These results may be explained by the fact that nursing is a universal feminine profession especially in our society culture as well as 
the enrolment of the male students in this profession was started in the late decades. These findings agreed with (Selman et al., 2018) who conducted a study on critical care nurses and they indicates that the female is the dominant gender among the current study, in which $73.3 \%$ of nurses in the study group, Also, results validated by (Rajeswaran et al., 2018) who stated that the majority of the study sample $(70 \%)$ were female. Also, results supported by (Shah et al., 2019)

Concerning to educational level; the present study findings demonstrated that, more than half of the study sample graduated from a technical institute. This may be explained by a little number of faculty's graduates had employed in the university hospital and other like to work in schools or ministry of health hospital. This study results is similar to the study done by (Eubayd \& Abbas, 2017) that stated the highest percentage related to the educational levels is seen to be educated in the nursing clinical institute that are $40 \%$ in the study group. This result was contradicting with ( Al-Ani et al., 2014) they found that most of participants $(41.2 \%)$ held a diploma degree in nursing.

Study results displayed that, more than half of the studied groups had less than five years of experience in working at critical units because they were newly graduated and young aged. Studied results were supported by ( Al-Ani et al., 2014) who founded that more than half (56.3\%) of nurses have less than five years of critical care units' experience while only (33.7\%) have more than five years of experience in working at ICU. These results contradicting with (Rajeswaran et al., 2018) they stated that The duration of work experience was divided between $49.3 \%$ (working from between 1 and 6 years) and 50.6\% (working for more than 7 years). Study findings illustrated that the most of the study sample had not attended any previous workshop regarding cardio pulmonary resuscitation process (CPR). This may be due to negligence from in-service educational training unit in the hospital about importance of this topic, no preparatory program for new staff and lack of interest from critical care nurses to attend any conferences or workshops. These study findings agreed with ( Al-Ani et al., 2014), they illustrated that more than half $(61.2 \%)$ of critical care nurses had no previous workshop attendance regarding cardiopulmonary resuscitation.

Regarding overall total studied samples' knowledge Score levels about CPR process, the results showed that, more than two third of studied sample had unsatisfactory knowledge during pre education 'while $93.3 \%$ of them had satisfactory knowledge level post three months test, these results confirmed by (Tíscar-González et al., 2019) they founded that the nurses level of knowledge improved post educational program, moreover validation by ( Rajeswaran et al., 2018) they stated that the majority of studied samples' knowledge score $88.6 \%$ post 12 week of test. Further validation by (Selman \& Ahmed (2018) they reached to that the overall assessment of nurses' knowledge in the study group showing a fair knowledge towards cardiopulmonary resuscitation process during the pre-test period $(86.7 \%)$, and their knowledge is increased to a good level during the post-test period $(100 \%)$ after receiving an educational program.

Regarding overall total practices levels of studied samples' roles in resuscitation Process pre\&post education regard CPR process, the results showed that, the outcome of post-test of CPR knowledge and skills of nurses post educational protocol improved considerably post intervention given that there was no significant previous training or attendance any workshop.

These results confirmed by (Munezero et al., 2018) they conducted study that concluded that increase of nurses' knowledge \& skills after training, from a pretest average scores of $46.0 \%$ to $81.3 \%$ for skills and $53.8 \%$ to $82.45 \%$ for knowledge. As well as, (Rajeswaran \& Ehlers (2014) stated that the results of the CPR skills tests showed lower scores before training was $32.4 \%$ indicating a low level of competence while post-test three months reach to high level of competence (76.2). Moreover, (Elazazay et al., 2012

Regarding Correlations between total knowledge score and total practice score among study sample pre\& post educational protocol (three months) results revealed that there was highly statistical significance between total knowledge and practice score at pre \& post education (three months). These results were disagreed with (Elazazay et al., 2012) who stated that decreasing knowledge level at the initial baseline data assessment for nurses in ICU that also reflected in the nurses' practice at pre-intervention of the program, that converted to a significant improvement of both knowledge and practice after intervention.

Regarding Correlations between personal data of the studied groups and total practice score pre-education and post-education (three months), results revealed that, there was no statistically significant correlation between all socio-demographic characteristics for study group and their total practice score pre and post-educational protocol except in a study sample's age it had a significant correlation with mean practice score after three months post education with $\mathrm{p}$-value (.052). These results similar to (Rajeswaran \& Ehlers (2014) they demonstrated that their no significant differences in terms of gender, age and experience, although a study conducted in Belgium demonstrated that accumulated work experience was associated with improved CPR skills. On other hand, study results contraindicated with ( Rajeswaran et al., 2018) they founded that Male participants scored slightly better than their female counterparts $(52.9 \%$ vs. $46.1 \%)$ in the pre-test, also, The highest improvement realised (28.5\%) was observed among the participants with 5-6 years of experience and the least improvement $(22.7 \%)$ was among the participants who have more than 11 years of experience

\section{Recommendations}

1. Greater attention and closely observation from hospital on nurses' performance during resuscitation process.

2. Training program should be arranged for nurses working in critical care units about CPR and receive regular, periodic CPR courses, updating nurses on the latest CPR techniques, technologies and developments.

3. The hospital organization must be emphasize to develop Mock Code Blue system and it' $s$ team for resuscitation at emergency situations when needed.

4. Future studies have to be carried out in order to assess factors associated with poor level of practice regarding resuscitation process pre-educational protocol. 
Acknowledgment

The researchers would like to acknowledge the contribution of all participants who kindly agreed to take part in the study. They generously gave their time and attention to conduct this study. This study would have been impossible without their generosity.

\section{References}

1. Al-Ani, B. A. J., \& Al, M. A. A. K. M. (2014). Assessment of Nurses' Knowledge towards Cardiopulmonary Resuscitation at Al-Najaf City's Teaching Hospital. kufa Journal for Nursing sciences, 4(1), 208-217.

2. American Heart Association, based on scientific research and American Heart Association guidelines, Mar 31, 2012

3. Burkle, C. M., Swetz, K. M., Armstrong, M. H., \& Keegan, M. T. (2013). Patient and doctor attitudes and beliefs concerning perioperative do not resuscitate orders: anesthesiologists' growing compliance with patient autonomy and self determination guidelines. $B M C$ anesthesiology, 13(1), 2

4. Cole, J. B., Knack, S. K., Karl, E. R., Horton, G. B., Satpathy, R., \& Driver, B. E. (2019). Human Errors and Adverse Hemodynamic Events Related to "Push Dose Pressors" in the Emergency Department. Journal of Medical Toxicology, 15(4), 276-286.

5. Elazazay, H. M., Abdelazez, A. L., \& Elsaie, O. A. (2012). Effect of cardiopulmonary resuscitation training program on nurses knowledge and practice. Life Sci J, 9(4), 3494-3503.

6. Eubayd, Ali \& Abbas, Sabah. (2017). Effectiveness of an Educational Program on Nurses Knowledge Concerning Cardiopulmonary Resuscitation at lmam Hussein Medical City in Holy Kerbala'a Governorate. Journal of Integrated Health Science. 6. 10.9790/1959-0604047175.

7. Hasegawa, K., et al. (2013). "Association of prehospital advanced airway management with neurologic outcome and survival in patients with out-of-hospital cardiac arrest." Jama 309(3): 257-266.

8. Hasselqvist-Ax, I., Riva, G., Herlitz, J., Rosenqvist, M., Hollenberg, J., Nordberg, P., ... \& Karlsson, T. (2015). Early cardiopulmonary resuscitation in out-of-hospital cardiac arrest. New England Journal of Medicine, 372(24), 2307-2315

9. Kerrey, Benjamin \& Rinderknecht, Andrea \& Mittiga, Matthew. (2017). High Risk, Low Frequency: Optimizing Performance of Emergency Intubation for Children. Annals of Emergency Medicine. 70. 10.1016/j.annemergmed.2017.06.015.

10. Lo, Zhiwen. (2016). An Evaluation of the Technical and Non-technical Skills of Emergency Teams during Adult Cardiopulmonary Resuscitation in the Emergency Department. 10.13140/RG.2.1.3121.5122.

11. Mansouri, A., Ahmadvand, A., Hadjibabaie, M., Javadi, M., Khoee, S. H., Dastan, F., \& Gholami, K. (2014). A review of medication errors in iran: sources, underreporting reasons and preventive measures. Iranian journal of pharmaceutical research: IJPR, 13(1), 3.

12. Parajulee S, Selvaraj V. Knowledge of nurses towards cardiopulmonary resuscitation in a tertiary care teaching hospital in Nepal. Journal of Clinical and Diagnostic Research, 2011; 5(8): 1585- 1588.

13. Rajeswaran, L., \& Ehlers, V. J. (2014). Cardiopulmonary resuscitation knowledge and skills of registered nurses in Botswana. curationis, 37(1), 1-7.

14. Rajeswaran, L., Cox, M., Moeng, S., \& Tsima, B. M. (2018). Assessment of nurses' cardiopulmonary resuscitation knowledge and skills within three district hospitals in Botswana. African journal of primary health care \& family medicine, 10(1), 1-6.

15. Ray, M. A. (2016). Transcultural caring dynamics in nursing and health care. FA Davis.

16. Ray, M. A. (2016). Transcultural caring dynamics in nursing and health care. FA Davis.

17. Rinderknecht, A. S., Dyas, J. R., Kerrey, B. T., Geis, G. L., Ho, M. H., \& Mittiga, M. R. (2017). Studying the safety and performance of rapid sequence intubation: data collection method matters. Academic Emergency Medicine, 24(4), 411-421.

18. Selman, F. K., \& Ahmed, S. A. (2018). Effectiveness of an Educational Program on Nurses' Practices Concerning Nursing Interventions for Patients with Coronary Artery Disease in AL-Nasiriyah Heart Center. Indian Journal of Public Health Research \& Development, 9(8).

19. Shah M, Jan O, Hussain A, Gul T, Naila N (2019) Knowledge and Practice of Nurses Regarding CPR in Private Tertiary Care Hospital Peshawar, KP, Pakistan. J Nurs Care 8:475. DOI: 10.4172/2167-1168.1000475

20. Simmes, F. M., Schoonhoven, L., Mintjes, J., Fikkers, B. G., \& van der Hoeven, J. G. (2012). Incidence of cardiac arrests and unexpected deaths in surgical patients before and after implementation of a rapid response system. Annals of intensive care, 2(1), 20.

21. Terzi, A. B. (2012). Nurse's role in the modern resuscitation era. Hospital Chronicles, 7(1), 25-31.

22. Tíscar-González, V., Blanco-Blanco, J., Gea-Sánchez, M., Molinuevo, A. R., \& Moreno-Casbas, T. (2019). Nursing knowledge of and attitude in cardiopulmonary arrest: cross-sectional survey analysis. PeerJ, 7, e6410.

23. Waldron, N., Johnson, C. E., Saul, P., Waldron, H., Chong, J. C., Hill, A. M., \& Hayes, B. (2016). Development of a video-based education and process change intervention to improve advance cardiopulmonary resuscitation decision-making. BMC health services research, 16(1), 555

24. Wolfe, H., Zebuhr, C., Topjian, A. A., Nishisaki, A., Niles, D. E., Meaney, P. A., ... \& Apkon, M. (2014). Interdisciplinary ICU cardiac arrest debriefing improves survival outcomes. Critical care medicine, 42(7), 1688.

25. Yang, C. L., Wen, J., Li, Y. P., \& Shi, Y. K. (2012). Cardiocerebral resuscitation vs cardiopulmonary resuscitation for cardiac arrest: a systematic review. The American journal of emergency medicine, 30(5), 784-793. 